\title{
CORRELAÇÕES DA ALTURA E DIÂMETRO COM TENSÕES DE CRESCIMENTO EM ÁRVORES DE Corymbia citriodora E Eucalyptus urophylla
}

\author{
Alexandre Monteiro Carvalho², Maria da Penha Moreira Gonçalves ${ }^{3}$, Kelysson de Freitas Amparado ${ }^{4}$, \\ João Vicente de Figueiredo Latorraca² e Rosilei Aparecida Garcia²
}

\begin{abstract}
RESUMO - O uso múltiplo das florestas plantadas pelo setor industrial de base florestal vem aumentando gradativamente. Hoje, a utilização da madeira de eucalipto para fins mais nobres já é uma realidade. Porém, algumas limitações como a presença de tensões de crescimento são responsáveis por grandes perdas no setor. As tensões de crescimento geram rachaduras de topo em toras e tábuas e também empenamentos nas peças após o desdobro. Dentro desse contexto, este trabalho objetivou avaliar essas tensões em diferentes classes diamétricas e alturas, em indivíduos de Corymbia citriodora e Eucalyptus urophylla, usando o método não destrutivo da medição da deformação residual longitudinal (DRL), ao redor da circunferência dos troncos das árvores. As deformações residuais longitudinais, associadas às tensões de crescimento para Corymbia citriodora e Eucalyptus urophylla, respectivamente, foram, em média, de 0,106 e 0,092 mm. O efeito da classe diamétrica em Corymbia citriodora evidenciou correlação negativa significativa com a DRL, enquanto a altura não mostrou qualquer tendência quando relacionada com a DRL dessa espécie. Em Eucalyptus urophylla foi possível observar que tanto a correlação com o diâmetro à altura do peito (DAP) quanto com a altura foi significativa, apresentando coeficientes negativos, ou seja, as tensões de crescimento foram menores em árvores com maiores DAP e maiores alturas.
\end{abstract}

Palavras-chave: Eucalipto, Deformação residual longitudinal e Tensões de crescimento.

\section{HEIGHT AND DIAMETER CORRELATIONS WITH GROWTH TENSIONS IN TREES OF Corymbia citriodora AND Eucalyptus urophylla}

\begin{abstract}
The multiple-use of planted forests by the forest products industrial sector has been increased gradually. Today, eucalyptus wood is used for nobler applications. However, some limitations, such as the presence of growth stresses, are responsible for great losses in the sector. Growth stresses generate cracks in the tops of logs and boards followed by warping. In this context, the objectives of the present work were to evaluate the growth stresses in individual Corymbia citriodora and Eucalyptus urophylla with different diameter and height classes, by the longitudinal residual strain (LRS) nondestructive method, around the trees circumference. The average longitudinal residual strain, associated to the Corymbia citriodora and Eucalyptus urophylla growth stresses, were 0.106 and $0.092 \mathrm{~mm}$, respectively. For Corymbia citriodora, the diameter at breast height (DBH) had a negative significant correlation with the LRS, while the height did not show a significant correlation. For Eucalyptus urophylla, a negative significant correlation between LRS and both variables, DBH and height, was observed. Indeed, growth stresses were lower for greater DBH and heights.
\end{abstract}

Keywords: Eucalyptus, Longitudinal residual deformation and Growth tensions.

\footnotetext{
${ }^{1}$ Recebido em 04.04.2008 e aceito para publicação em 14.10.2009.

${ }^{2}$ Departamento de Produtos Florestais, Instituto de Florestas, Universidade Federal Rural do Rio de Janeiro. E-mail: <amcarvalho@ufrrj.br>,<latorraca@ufrrj.br>e <rosileigar@ufrrj.br>.

${ }^{3}$ Graduanda em Engenharia Florestal, Departamento de Produtos Florestais, Instituto de Florestas, Universidade Federal Rural do Rio de Janeiro. E-mail: <penhaengflo@bol.com.br>.

${ }^{4}$ Mestrando em Ciências Ambientais e Florestais, Departamento de Produtos Florestais, Instituto de Florestas, Universidade Federal Rural do Rio de Janeiro. E-mail: <kelyssonfloresta@yahoo.com.br>.
} 


\section{INTRODUÇÃO}

Espécies florestais de rápido crescimento, como as do gênero Eucalyptus, são potenciais substitutos da madeira oriunda de florestas naturais para a obtenção de produtos sólidos, como os gerados em serrarias e utilizados em marcenarias e carpintarias. Inúmeros são os atributos que tornam importante o gênero Eucalyptus como fonte de matéria-prima fabril, por exemplo a sua capacidade produtiva, a adaptabilidade a diversos ambientes e, sobretudo, a diversidade de espécies, o que possibilita ao gênero atender a requisitos tecnológicos dos mais variados segmentos da produção industrial madeireira (ASSIS, 1999).

Os atributos de caráter silvicultural geralmente caminham em direção contrária em relação aos de cunho tecnológico. É comum uma espécie de elevada capacidade produtiva não ser adequada para dado tipo de uso ou, mesmo, ter utilidade restrita. Mesmo assim, a madeira de eucalipto vem, dia a dia, ganhando espaço na indústria de madeira serrada e produtos à base de madeira, diversificando sua utilização, até pouco tempo atrás prioritariamente destinada à indústria de celulose. Atualmente, já é uma realidade a produção de painéis aglomerados, chapas de fibras, compensados e madeira serrada de eucalipto. Porém, o uso da madeira serrada de eucalipto é limitado por algumas características indesejáveis, como a presença de tensões de crescimento.

Conforme comentaram Lima et al. (2004), as tensões de crescimento são forças que agem naturalmente sobre os tecidos das árvores, de forma a mantê-las íntegras e eretas, garantindo resistência à ruptura mecânica e à flexão no tronco, gerando resistência contra a incidência de ventos. Essas tensões nas partes mais externas dos fustes fazem o papel de armadura de aço nas colunas de concreto, sendo fundamentais para que os fustes das árvores não se quebrem facilmente quando submetidas a esforços laterais.

A origem das tensões de crescimento está na camada cambial dos troncos das árvores. Elas são geradas na madeira, devido às deposições consecutivas das novas camadas de células a partir do câmbio, durante o desenvolvimento da planta. A tendência dessas novas células é a expansão lateral enquanto, ao mesmo tempo, se contraem no sentido longitudinal. A adição de novas camadas de células ao tronco causa estado de tensão externa (tração). Tais tensões são distribuídas pelo tronco da árvore de forma cumulativa; isso devido à sucessiva deposição de camadas e respectivas tensões. Todas essas interações resultam no surgimento de forças de compressão compensatórias na parte central do tronco (KAUMAN et al., 1995; KUBLER, 1987; MALAN, 1995; SHIELD, 1995).

Os efeitos das tensões de crescimento podem ser observados nas toras após o abate das árvores e, principalmente, nas tábuas, durante as operações de desdobro em serraria. O resultado dessas tensões reflete-se na diminuição do rendimento em madeira serrada que, dependendo da sua magnitude, pode ocasionar grandes perdas durante as etapas da cadeia produtiva; isso devido à liberação dessas tensões, que causam defeitos como rachaduras e empenamentos. O mesmo acontece no processo de secagem das tábuas, quando se perde ainda mais material. Em determinadas circunstâncias, esses defeitos podem chegar a tornar inviável a entrada de madeira sólida de eucalipto no mercado de madeira serrada (LISBOA, 1993; TEJADA et al., 1997; LIMA et al., 2004).

Aas limitações à utilização da madeira de eucalipto devido à presença de tensões são mais notáveis em florestas jovens do que em adultas como consequência de diversos fatores, como a proporção de lenho juvenil no tronco. As causas das altas tensões de crescimento ainda não são totalmente conhecidas, mas há suspeitas de que estejam relacionadas, além da idade, a fatores genéticos, tamanho da tora, taxa de crescimento e inclinação do fuste. O melhoramento florestal pode fornecer uma solução para possibilitar a redução dos altos níveis de tensões, devidoao fato de ser esse um fenômeno geneticamente herdável (MALAN, 1995; SCHACHT et al., 1998).

Lima et al. (2004) destacaram que a intensidade das tensões de crescimento é altamente variável entre espécies, entre árvores da mesma espécie e também em diferentes posições dentro da árvore. De acordo com a distribuição das tensões no interior do tronco, peças de madeira removidas da periferia do tronco tenderam a diminuir seu comprimento em resposta a seu estado de tração, já as peças removidas próximas à medula tenderão a alongar-se em resposta ao seu estado de compressão.

A seleção de material com menores níveis de tensão de crescimento e a associação dessas tensões a características facilmente mensuráveis devem ser priorizadas nos programas de melhoramento genético florestal. Para isso, há a necessidade da avaliação dos níveis dessas tensões no tronco das árvores ainda vivas. 
Dentro desse contexto, este trabalho objetivou avaliar tensões de crescimento em diferentes classes diamétricas e alturas, em Corymbia citriodora e Eucalyptus urophylla, através do método não destrutivo da medição da deformação residual longitudinal (DRL), ao redor da circunferência dos troncos de árvores vivas.

\section{MATERIAL E MÉTODOS}

\subsection{Material}

O material de estudo foi amostrado em plantios de Corymbia citriodora e Eucalyptus urophylla, ambos com 15 anos de idade, implantados com espaçamento de $36 \mathrm{~m}$ entre linhas e $2 \mathrm{~m}$ entre árvores, em povoamentos com aproximadamente 250 ha, localizados no Município de Seropédica-RJ.

Os plantios situavam-se em zona de baixada, a $22^{\circ} 49^{\prime} \mathrm{S}$ e $43^{\circ} 38^{\prime} \mathrm{W}$, apresentando altitude média de $30 \mathrm{~m}$, com relevo praticamente plano e ondulações de 0 a $3^{\circ}$ de declive, clima tropical chuvoso de inverno seco, tipo climático $A w$, com verão úmido e inverno seco. A temperatura média do mês mais frio foi superior a $18{ }^{\circ} \mathrm{C}$, a diferença entre a temperatura média do mês mais quente e a do mês mais frio foi inferior a $12{ }^{\circ} \mathrm{C}$ e a precipitação média anual ficou entre 1.000 e $1.500 \mathrm{~mm}$, com o mês mais seco apresentando menos de 40 mm de precipitação.

Foram avaliadas 60 árvores, sendo 30 de cada espécie. As árvores foram selecionadas aleatoriamente nos povoamentos, tendo-se o cuidado de escolher as mais representativas de acordo com a classe diamétrica. Não foram consideradas as árvores de bordadura e as que apresentavam sintomas de doenças.

\subsection{Avaliações realizadas nas árvores}

Foi utilizado como equipamento de medição o extensômetro (medidor de deformações de crescimento), para obtenção da Deformação Residual Longitudinal (DRL) e avaliação das tensões de crescimento nas árvores (Figura 1). O aparelho verifica a deformação em mm, originada na periferia do tronco sem casca, após a liberação das tensões, através de pequena perfuração feita com arco de pua com broca de $20 \mathrm{~mm}$, que provoca o rompimento dos tecidos do lenho. Essa perfuração é feita até uma profundidade suficiente para que seja obtida a estabilização da leitura no relógio do aparelho, em torno de $2,5 \mathrm{~cm}$.

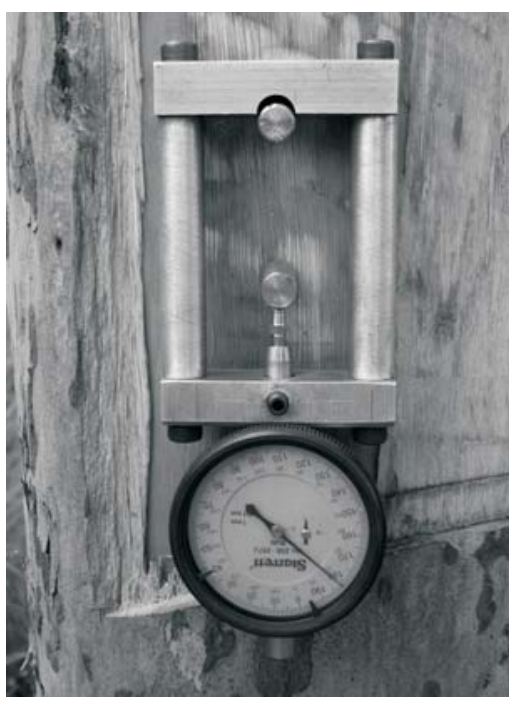

Figura 1 - Equipamento de medição da DRL. Figure 1 - Equipment for LRS measurement.

A perfuração é feita após a fixação de dois pinos metálicos, que são cravados em um painel aberto no tronco, depois de retirada da casca. Os pinos são fixados na madeira a uma distância de $45 \mathrm{~mm}$ um do outro, na direção da grã. A perfuração pela broca ocorre entre os pinos, fazendo-se com que eles se aproximem. A medição da deformação é feita logo após a furação e estabilização do relógio.

As verificações foram realizadas em três repetições ao longo da circunferência da árvore, na altura do Diâmetro à Altura do Peito (DAP), aproximadamente 1,30 m a partir da base da árvore. Os diâmetros de todas as árvores selecionadas foram medidos com fita diamétrica, e as alturas, com o hipsômetro Blume-leiss.

\subsection{Análise dos resultados}

Para a avaliação dos resultados, os dados foram agrupados em seis classes de diâmetro, com amplitude de $4,1 \mathrm{~cm}$. As alturas do Corymbia citriodora foram distribuídas em cinco classes com amplitude de $4 \mathrm{~m}$, e as alturas do Eucalyptus urophylla o foram dentro de quatro classes também com amplitude de $4 \mathrm{~m}$.

Foi realizado o estudo das associações das DRL com os DAP e das DRL com as alturas totais, sendo determinada a correlação linear simples. Para verificação e análise da magnitude das variações entre as DRL foram calculados coeficientes de variação experimental.

R. Árvore, Viçosa-MG, v.34, n.2, p.323-331, 2010 


\section{RESULTADOS E DISCUSSÃO}

Na Tabela 1 são apresentados os valores médios da deformação residual longitudinal (DRL) por classe diamétrica de Corymbia citriodora e Eucalyptus urophylla e os respectivos coeficientes de variação.

Na Tabela 2, a deformação residual longitudinal (DRL) está apresentada de acordo com a classe de altura das árvores amostradas no trabalho.

Os coeficientes de variação encontrados da Deformação Residual Longitudinal no Corymbia citriodora foram considerados medianos, demonstrando variabilidade considerável dos valores de DRL dentro das classes, sendo valores semelhantes observados em Eucalyptus dunnii na idade de 13 anos e nos clones de Eucalyptus spp na idade de 10 anos (TRUGILHO et al., 2004; TRUGILHO et al., 2006).
Na Tabela 1, verifica-se que em Corymbia citriodora foi observada menor variação dentro da classe diamétrica 6 (12\%) e maior na classe diamétrica 3 (30\%). As menores variações (classes diamétricas 5 e 6 ) podem estar ligadas ao fato de que as frequências dentro dessas classes foram muito baixas, sendo encontradas apenas três árvores na classe 5 e duas árvores na classe 6. Esses valores de coeficiente de variação indicam que se deve aumentar o tamanho da amostra avaliada, ou seja, o número de árvores por classes.

Nessa espécie, o coeficiente de correlação simples (r) entre o valor da DRL média e o DAP médio foi de $-0,84$, sendo identificada, assim, interação significativamente negativa, indicativa de que, no geral, árvores de maior incremento diamétrico apresentam menores valores de DRL nessa espécie com essa idade.

Tabela 1 - Deformação residual longitudinal - DRL (mm) por classe diamétrica de Corymbia citriodora e Eucalyptus urophylla. Table 1- Longitudinal residual strain - (LRS) ( $\mathrm{mm}$ ) by diametric class of Corymbia citriodora and Eucalyptus urophylla.

\begin{tabular}{|c|c|c|c|c|c|c|c|c|c|c|c|c|c|c|c|}
\hline \multicolumn{7}{|c|}{ Corymbia citriodora } & \multicolumn{9}{|c|}{ Eucalyptus urophylla } \\
\hline$\overline{\text { Classe }}$ & DAP & DAP & Freq & . Centro & DAP & DRL & $\mathrm{CV}$ & Classe & DAP & DAP & Freq & Centro & DAP & DRL & $\mathrm{CV}$ \\
\hline DAP & $\begin{array}{l}\text { inferior } \\
(\mathrm{cm})\end{array}$ & $\begin{array}{c}\text { superior } \\
(\mathrm{cm})\end{array}$ & & $\begin{array}{c}\text { Classe } \\
(\mathrm{cm})\end{array}$ & $\begin{array}{c}\text { médio } \\
(\mathrm{cm})\end{array}$ & $\begin{array}{l}\text { média } \\
(\mathrm{mm})\end{array}$ & (\%) & DAP & $\begin{array}{l}\text { inferior } \\
(\mathrm{cm})\end{array}$ & $\begin{array}{l}\text { superior } \\
(\mathrm{cm})\end{array}$ & & $\begin{array}{c}\text { Classe } \\
(\mathrm{cm})\end{array}$ & $\begin{array}{l}\text { médio } \\
\text { (cm) }\end{array}$ & $\begin{array}{l}\text { média } \\
(\mathrm{mm})\end{array}$ & (\%) \\
\hline 1 & 24,3 & 28,4 & 5 & 26,35 & 25,62 & 0,129 & 25,19 & 1 & 24,3 & 28,4 & 5 & 26,35 & 26,32 & 0,106 & 22,18 \\
\hline 2 & 28,4 & 32,5 & 6 & 30,45 & 30,10 & 0,099 & 24,75 & 2 & 28,4 & 32,5 & 7 & 30,45 & 29,64 & 0,095 & 26,42 \\
\hline 3 & 32,5 & 36,6 & 6 & 34,55 & 34,66 & 0,109 & 30,43 & 3 & 32,5 & 36,6 & 5 & 34,55 & 33,81 & 0,103 & 48,86 \\
\hline 4 & 36,6 & 40,7 & 8 & 38,65 & 38,69 & 0,109 & 24,39 & 4 & 36,6 & 40,7 & 5 & 38,65 & 37,72 & 0,075 & 42,63 \\
\hline 5 & 40,7 & 44,8 & 3 & 42,75 & 42,49 & 0,083 & 13,85 & 5 & 40,7 & 44,8 & 5 & 42,75 & 41,64 & 0,086 & 22,63 \\
\hline 6 & 44,8 & 48,9 & 2 & 46,85 & 46,89 & 0,081 & 12,22 & 6 & 44,8 & 48,9 & 3 & 46,85 & 48,14 & 0,079 & 23,82 \\
\hline
\end{tabular}

Freq. = frequência em $\mathrm{n}^{\circ}$ de indivíduos.

$\mathrm{CV} \%$ = coeficiente de variação experimental.

Freq. $=$ frequency in number of individuals.

$\mathrm{CV} \%$ = experimental coefficient variation.

Tabela 2-Deformação residual longitudinal ( $\mathrm{mm}$ ) por classe de altura de Corymbia citriodora e Eucalyptus urophylla. Table 2 - Longitudinal residual strain - (LRS) $(\mathrm{mm})$ by height class of Corymbia citriodora e Eucalyptus urophylla.

\begin{tabular}{|c|c|c|c|c|c|c|c|c|c|c|c|c|c|c|c|}
\hline \multicolumn{8}{|c|}{ Corymbia citriodora } & \multicolumn{8}{|c|}{ Eucalyptus urophylla } \\
\hline $\begin{array}{c}\text { Classe } \\
\mathrm{H}\end{array}$ & $\begin{array}{c}\mathrm{H} \\
\text { inferior } \\
(\mathrm{m})\end{array}$ & $\begin{array}{c}\mathrm{H} \\
\text { superior } \\
(\mathrm{m})\end{array}$ & Freq & $\begin{array}{c}\text { Centro } \\
\text { Classe } \\
(\mathrm{m})\end{array}$ & $\begin{array}{c}\mathrm{H} \\
\text { médio } \\
(\mathrm{m})\end{array}$ & $\begin{array}{c}\text { DRL } \\
\text { média } \\
(\mathrm{mm})\end{array}$ & $\begin{array}{l}\text { CV } \\
(\%)\end{array}$ & $\begin{array}{c}\text { Classe } \\
\mathrm{H}\end{array}$ & $\begin{array}{c}\mathrm{H} \\
\text { inferior } \\
(\mathrm{m})\end{array}$ & $\begin{array}{c}\mathrm{H} \\
\text { superior } \\
(\mathrm{m})\end{array}$ & Freq & $\begin{array}{c}\text { Centro } \\
\text { Classe } \\
(\mathrm{m})\end{array}$ & $\begin{array}{c}\mathrm{H} \\
\text { médio } \\
(\mathrm{m})\end{array}$ & $\begin{array}{c}\text { DRL } \\
\text { média } \\
(\mathrm{mm})\end{array}$ & $\begin{array}{l}\text { CV } \\
(\%)\end{array}$ \\
\hline 1 & 29 & 33 & 4 & 31,0 & 32,02 & 0,123 & 28,53 & 1 & 27 & 31 & 5 & 29 & 28,90 & 0,101 & 20,64 \\
\hline 2 & 33 & 36 & 6 & 34,5 & 34,90 & 0,101 & 26,28 & 2 & 31 & 35 & 12 & 33 & 32,70 & 0,099 & 37,04 \\
\hline 3 & 36 & 39 & 6 & 37,5 & 36,93 & 0,107 & 23,33 & 3 & 35 & 39 & 8 & 37 & 36,92 & 0,078 & 34,48 \\
\hline 4 & 39 & 42 & 12 & 40,5 & 40,95 & 0,100 & 30,25 & 4 & 39 & 43 & 5 & 41 & 40,16 & 0,087 & 25,95 \\
\hline 5 & 42 & 45 & 2 & 43,5 & 43,10 & 0,16 & 34,13 & - & - & - & - & - & - & - & - \\
\hline
\end{tabular}

$\mathrm{H}=$ altura.

$\mathrm{H}=$ Height

Freq. = frequência em número de indivíduos.

CV\% = coeficiente de variação experimental.

Freq. $=$ frequency in number of individuals.

$\mathrm{CV} \%=$ experimental coefficient variation.

R. Árvore, Viçosa-MG, v.34, n.2, p.323-331, 2010 
Muneri et al. (2000) encontraram correlação positiva e significativa de DRL com a densidade do alburno $(\mathrm{r}=0,40)$ com o DAP $(\mathrm{r}=0,51)$ com a altura da árvore $(r=0,44)$ e com o encurvamento da madeira serrada $(\mathrm{r}=0,67)$ de Eucalyptus cloeziana, indicando, assim, que, além das propriedades estudadas, outras podem causar interferências nas intensidades das tensões de crescimento no gênero Eucalyptus.

No Corymbia citriodora, a deformação residual longitudinal média total foi de 0,106 mm, sendo esse um valor maior que o normalmente observado no gênero Eucalyptus.

Lima et al. (2004) encontraram valor de 0,071 mm em cinco materiais genéticos cultivados experimentalmente no Espírito Santo. Cardoso Júnior et al. (2005) verificaram 0,065 mm em clones de híbridos de Eucalyptus. Essas diferenças estão relacionadas, principalmente, com a espécie, a idade de medição e o local ou sítio.

Esses mesmos autores apontaram a intensidade de desbastes como um dos fatores decisivos na intensidade das tensões de crescimento em uma mesma classe diamétrica, influenciando significativamente os índices de rachadura de extremidade de toras e o encurvamento da peça serrada úmida. Esse fato pode ser explicado partindo-se do princípio de que, quanto maior a precocidade e quantidade de desbastes, menor a competição entre as árvores, permitindo, portanto, que as árvores cresçam livremente e em ritmo acelerado, e pruduzam madeira mais homogênea e estável. Esse fator pode ter sido decisivo no estabelecimento das tensões do povoamento de Corymbia citriodora estudado neste trabalho, já que, como esse povoamento foi implantado para a produção de carvão, não houve preocupação com a execução de práticas silviculturais como desbaste, o que acentuou a competição entre as árvores.

Ainda de acordo com a Tabela 1, os coeficientes de variação da DRL nas diferentes classes diamétricas de Eucalyptus urophylla foram no geral medianos, assim como observado anteriormente em Corymbia citriodora. Porém, os valores referentes às classes diamétricas 3 e 4 se mostraram elevados (49\% e 43\%, respectivamente), denotando maior variabilidade da DRL dentro dessas classes. Esse fator pode ser considerado negativo, já que levará a um maior desvio da média obtida dos valores referentes às tensões de crescimento, sendo necessário um maior número de árvores estudadas dentro das classes. A menor variação ocorreu na classe diamétrica 1 , compreendida entre 24,3 e 28,4 cm, denotando, assim, maior homogeneidade nos valores de tensões dentro dessa classe.

Com relação à altura (Tabela 2), assim como foi observado para o diâmetro, foi verificada a ocorrência de valores de coeficiente de variação medianos, o que indica que os valores de deformação residual longitudinal (DRL), em Corymbia citriodora tiveram variações consideráveis entre si, também necessitando de maior número de árvores por classe.

Ainda na Tabela 2 se pode observar que os coeficientes de variação, assim como as análises anteriores, se mantiveram no geral medianos nas quatro classes de alturas de Eucalyptus urophylla. Foram obtidas maiores variações na classe 2 , com coeficiente de variação de $37 \%$, e as menores na classe 1 , com $21 \%$. Porém, a classe 1 foi a que apresentou maior DRL média $(0,123 \mathrm{~mm})$.

Conforme descreveu Chafe (1979), a diferença de comportamento das tensões de crescimento entre espécies é normal, sugerindo que essas podem ser substancialmente controladas por fatores genéticos. Para esse autor, tal variação não existe somente entre espécies, mas também entre árvores de uma mesma espécie e até entre toras de uma mesma árvore.

Na Figura 2 foram inseridos os resultados das análises de correlação entre as deformações observadas (DRL) e as classes de diâmetro das árvores de Corymbia citriodora e de Eucalyptus urophylla.

Na Figura 2, mostra-se a tendência da diminuição da DRL em árvores de Corymbia citriodora com maiores diâmetros. Foi observado valor de 0,129 $\mathrm{mm}$ na classe diamétrica 1 , compreendida entre 24,3 e $28,4 \mathrm{~cm}$, onde foram encontrados os diâmetros menores, e valores de $0,081 \mathrm{~mm}$ na classe diamétrica 6 , com diâmetros compreendidos entre 44,8 e 48,9 cm, indicando redução da DRL média com o aumento da classe diamétrica. Esse resultado indica que árvores com maiores diâmetros tendem a possuir menores valores da DRL, estando em conformidade com o encontrado por Fernandes (1989) e Trugilho et al. (2004).

R. Árvore, Viçosa-MG, v.34, n.2, p.323-331, 2010 


\section{Classes de DAP x DRL em Corymbia citriodora e Eucalyptus urophylla}

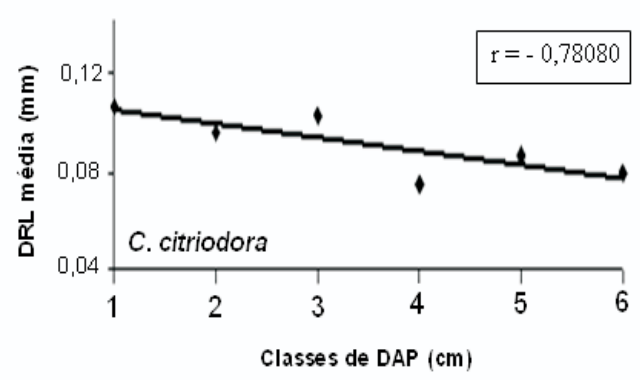

\begin{tabular}{|l|l|l|l|l|l|}
\hline 24,3 a 28,4 & 28,4 a 32,5 & 32,5 a 36,6 & 36,6 a 40,7 & 40,7 a 44,8 & 44,8 a 48,9 \\
\hline
\end{tabular}

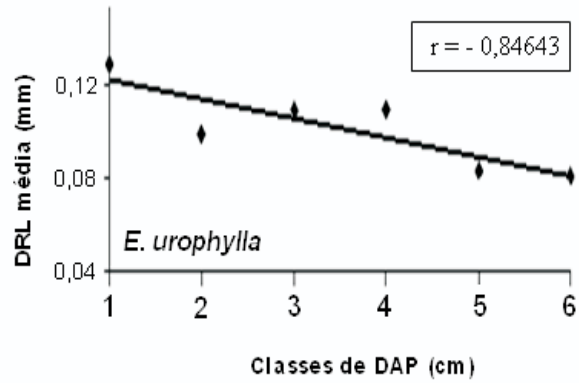

\begin{tabular}{|l|l|l|l|l|l|}
\hline 24,3 a 28,4 & 28,4 a 32,5 & 32,5 a 36,6 & 36,6 a 40,7 & 40,7 a 44,8 & 44,8 a 48,9 \\
\hline
\end{tabular}

Figura 2 - Comportamento da DRL em função da classe diamétrica de Corymbia citriodora e Eucalyptus urophylla. Figure 2-LRS behavior as a function of diametric class of Corymbia citriodora and Eucalyptus urophylla.

Em Eucalyptus dunnii nas idades de 8 e 13 anos, entretanto, não foi verificada correlação significativa entre a DRL e as características de crescimento por Trugilho et al. (2004), nas idades de 15 e 19 anos, e por autores como Souza (2002), podendo, então, essas relações ser influenciadas por diversos fatores.

Já para Lima et al. (2004) as características de crescimento de fácil medição são mais fortemente correlacionadas com as DRL do que com as propriedades da madeira, que são mais difíceis de serem dimensionadas.

Árvores individuais exibiram variação ao redor do tronco, denotando certa instabilidade ao longo da circunferência. Esse resultado pode estar associado a fatores ambientais predominantes, como a competição por luz ou pelo efeito de ventos, inclinação do fuste, pela variação do ângulo da grã na madeira, pelos fatores relacionados à presença de madeira de reação ou, mesmo, pela tortuosidade (SCHACHT et al., 1998). A variação pode estar associada ainda a fatores internos, como a constituição do material do tronco de lenho juvenil.

Na Figura 3, encontram-se os gráficos e coeficientes de correlação entre as deformações (DRL) e as alturas das árvores.

Pode-se observar, nessa figura, que não foi apresentada qualquer tendência quando relacionadas às classes de altura com a DRL. O valor de coeficiente de correlação linear simples encontrado foi igual a -0,2632, sendo esse não significativo. Essa característica de crescimento do Corymbia citriodora estudado não se mostrou variável a ser indicada para seleção de árvores com as tensões de crescimento desejadas. Resultados similares foram encontrados por Lima et al. (2004), observando que a altura total da árvore não resultou em correlação significativa com a DRL.

Nos resultados, foi possível observar que o coeficiente de correlação simples (r) verificado entre o diâmetro e a DRL de Eucalyptus urophylla foi de $-0,78$ e entre a DRL e altura, de $-0,78$, sendo ambos significativos e negativos, levando a constatação de que a DRL diminuiu à medida que o diâmetro e altura aumentaram.

Os valores de correlação obtidos de diâmetro estão de acordo com o trabalho de Wilkins e Kitahara (1991), que obtiveram forte correlação negativa com a mesma característica em Eucalyptus grandis com 12,5 e 22 anos $(r=-0,70)$.

Ao contrário do encontrado neste trabalho, Muneri et al. (2000) obtiveram correlações positivas e significativas da DRL com a altura e diâmetro das árvores de Eucalyptus cloeziana, possivelmente por terem estudado árvores de apenas 4 anos de idade, que apresentam comportamento diferenciado. Hillis e Brown (1978) comentaram que a madeira do gênero Eucalyptus, oriunda de árvores jovens, apresenta contrações excessivas, e sua utilização gera o 
Classes de altura x DRL em Corymbia citriodora e Eucalyptus urophylla
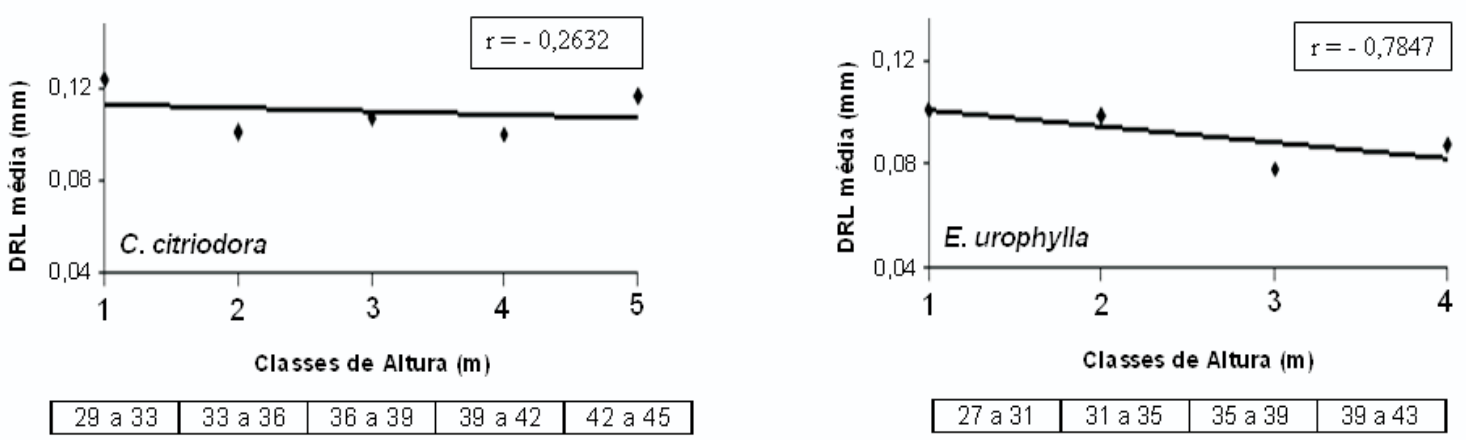

Figura 3 - Comportamento da DRL em função da classe de altura de Corymbia citriodora e Eucalyptus urophylla. Figure 3 - LRS behavior as a function of height class of Corymbia citriodora and Eucalyptus urophylla.

aparecimento de defeitos como rachaduras e empenamentos. Em seu trabalho, Ponce (1995) relataou que a madeira de eucalipto formada nos primeiros anos de idade da árvore, convencionalmente até cerca de 10 anos, apresenta fibras mais curtas, menor densidade e, consequentemente, propriedades mecânicas inferiores à madeira formada em idade mais elevada.

Muneri et al. (2000), juntamente com Lima et al. (2004), citaram ainda o fator de estabilidade como sendo altamente correlacionado com a DRL, sendo esta tanto maior quanto maior esse fator (árvores baixas e de grande diâmetro), evidenciando-se que árvores maiores têm níveis de tensões mais baixos. Tal fato denota a importância de se analisar cada caso, pois diferentes idades, condições de crescimento, espécies ou, mesmo, características genéticas podem influenciar nas características da madeira produzida.

\section{CONCLUSÕES}

- As deformações residuais longitudinais, associadas às tensões de crescimento em Corymbia citriodora e Eucalyptus urophylla, respectivamente, em média de 0,106 e 0,092 mm, se mostraram maiores que as médias encontradas em trabalhos anteriores.

- Existe elevada variabilidade no material estudado em ambas as espécies, indicando a possibilidade de seleção de material superior para uso como peças serradas.
- A correlação simples entre a DRL e o diâmetro médio da classe foi negativa e significativa em Corymbia citriodora e Eucalyptus urophylla, havend,o assim, forte tendência de diminuição da DRL com o aumento do diâmetro no material estudado.

- Houve correlação negativa e significativa entre a DRL e altura de Eucalyptus urophylla, porém esse fato não foi observado em Corymbia citriodora, evidenciando-se o comportamento distinto das duas espécies nessa associação.

- De acordo com o observado com o coeficiente de variação experimental, para esse tipo de estudo é indicado que seja avaliado o maior número possível de indivíduos por classes de diâmetro e altura para assim, possivelmente, obter menor variação entre os valores e consequentemente maior homogeneidade dos dados.

- Tanto em Corymbia citriodora quando em Eucalyptus urophylla a correlação da DRL com as classes de DAP se mostraram negativas e significativas, podendo ser, a classe de DAP, um parâmetro a ser utilizado em seleções de indivíduos com menores intensidades de tensões de crescimento dessas espécies nessa idade.

\section{AGRADECIMENTOS}

Os autores agradecem à FAPERJ - Fundação Carlos Chagas Filho de Amparo à Pesquisa do Estado do Rio de Janeiro pelos recursos recebidos para a realização deste trabalho.

R. Árvore, Viçosa-MG, v.34, n.2, p.323-331, 2010 


\section{REFERÊNCIAS}

ASSIS, T. F. Aspecto do melhoramento de Eucalyptus para obtenção de produtos sólidos de madeira. In: WORKSHOP: TÉCNICAS DE ABATE, PROCESSAMENTO E UTILIZAÇÃO DA MADEIRA DE EUCALIPTO, 1999, Viçosa, MG. Anais... Viçosa, MG: Universidade Federal de Viçosa/SIF/IEF, 1999. p.61-72.

CARDOSO JÚNIOR, A. A. et al. Deformação residual longitudinal em diferentes espaçamentos e idades em clone de híbrido de Eucalyptus. Cerne, v.11, n.3, p.218-224, 2005.

CHAFE, S. C. Growth stresses in trees. Australian Forest Research, v.9, p.203-223, 1979.

FERNANDES, P. S. Variações de densidade da madeira e suas relações com as tensões de crescimento em progênies de Eucalyptus urophylla. 1982. 96f. Dissertação (Mestrado em Ciência e Tecnologia de Madeiras) Escola Superior de Agricultura "Luiz de Queiroz”, Piracicaba, 1982.

HILLIS, W. E.; BROWN, A. G. (Eds.) Eucalypts for wood production. Melbourne: CSIRO, 1978. 434p.

KAUMAN, W. G.; GERARD, J.; JIQING, H. Processing of Eucalyptus. Commonwealth Forestry Review, v.7, n.2, p.147-154, 1995.

KUBLER, H. Growth stresses in trees and related wood properties. Forest Products

Abstracts, v.10, n.3, p.61-119, 1987.

LIMA, J. T. et al. Deformações residuais longitudinais decorrentes de tensões de crescimento em eucaliptos e suas associações com outras propriedades. Revista Árvore, v.28, n.1, p.107-116, 2004.

LIMA, I. L.; GARCIA, J. N.; NOGUEIRA, M. C. S. Influência do desbaste nas tensões de crescimento de Eucalyptus grandis Hill ex Maiden. Scientia Forestalis, n.58, p.111-125, 2000.

LISBOA, C. D. J. Estudo das tensões de crescimento em toras de Eucalyptus grandis Hill ex Maiden. 1993. 298f. Tese (Doutorado em Engenharia Florestal) Universidade Federal do Paraná, Curitiba, 1993.
MALAN, F. S. Eucalyptus improvement for lumber production. In: SEMINÁRIO INTERNACIONAL DE UTILIZAÇÃO DA MADEIRA DE EUCALYPTUS PARA SERRARIA, 1995, São Paulo. Anais... São Paulo: IPEF, IPT, IUFRO, LCF, ESALQ, USP, 1995. p.1-19.

MUNERI, A. et al. Relationships between surface longitudinal growth strain and tree size, wood properties and timber distortion of 4 years old plantation grown Eucalyptus cloeziana. In: IUFRO CONFERENCE - THE FUTURE OF EUCALYPTUS FOR WOOD PRODUCTS, 2000, Lauceston. Proceedings... Launceston: IUFRO, 2000. p.292-300.

PONCE, R. H. Madeira serrada de eucalipto: desafios e perspectivas. In: SEMINÁRIO INTERNACIONAL DE UTILIZAÇÃO DA MADEIRA DE EUCALIPTO PARA SERRARIA, 1995, São Paulo. Anais... São Paulo: IPEF, IPT, IUFRO, LCF, ESALQ, USP, 1995. p.50-58.

SCHACHT, L.; GARCIA, J. N.; VENCOVSKY, R. Variação genética de indicadores de tensão de crescimento em clones de Eucalyptus urophylla. Scientia Forestalis, n.54, p.55-68, 1998.

SHIELD, E. D. Plantation grown eucalypts: utilization for lumber and rotary veneers-primary conversion. In: SEMINÁRIO INTERNACIONAL DE UTILIZAÇÃO DA MADEIRA DE EUCALIPTO PARA SERRARIA, 1995, São Paulo. Anais... São Paulo: IPEF, IPT, IUFRO, LCF, ESALQ, USP, 1995. p.133-139.

SOUZA, M. A. M. Deformação Residual Longitudinal (DRL) causada pelas tensões de crescimento em clones de híbridos de Eucalyptus. 2002. 72f. Dissertação (Mestrado em Ciência e Tecnologia da Madeira) - Universidade Federal de Lavras, Lavras, 2002.

TEJADA, A. et al. Reduction of growth stress in logs by direct heat treatment: assessment of a commercial-scale operation. Forest Products Journal, v.47, n.9, p.86-93, 1997.

TRUGILHO, P. F. et al. Deformação residual longitudinal (DRL) e tangencial (DRT) em seis clones de Eucalyptus spp. Cerne, v.12, n.3, p.279-286, 2006. 
TRUGILHO, P. F. et al. Efeitos da idade e classe diamétrica na deformação residual longitudinal em árvores de Eucalyptus dunnii Maiden. Revista Árvore, v.28, n.5, p. 235231, 2004.
WILKINS, A. P.; KITAHARA, R. S. Silvicultural treatments and associated growth rates, growth strains and wood properties in 12.5-year-old Eucalyptus grandis. Australian Forestry Journal, v.54, n.1, p.99-104, 1991. 